\title{
New Stability Tests of Positive Standard and Fractional Linear Systems
}

\author{
Tadeusz Kaczorek \\ Faculty of Electrical Engineering, Bialystok University of Technology, Bialystok, Poland \\ E-mail:kaczorek@isep.pw.edu.pl \\ Received March 1, 2011; revised June 8, 2011; accepted June 15, 2011
}

\begin{abstract}
New tests for checking asymptotic stability of positive 1D continuous-time and discrete-time linear systems without and with delays and of positive 2D linear systems described by the general and the Roesser models are proposed. Checking of the asymptotic stability of positive 2D linear systems is reduced to checking of suitable corresponding $1 \mathrm{D}$ positive linear systems. It is shown that the stability tests can be also applied to checking the asymptotic stability of fractional discrete-time linear systems with delays. Effectiveness of the tests is shown on numerical examples.
\end{abstract}

Keywords: Fractional, Positive, Linear, System, Asymptotic Stability, Test

\section{Introduction}

A dynamical system is called positive if its trajectory starting from any nonnegative initial state remains forever in the positive orthant for all nonnegative inputs. An overview of state of the art in positive theory is given in the monographs $[1,2]$. Variety of models having positive behavior can be found in engineering, economics, social sciences, biology and medicine, etc.

New stability conditions for discrete-time linear systems have been proposed by M. Busłowicz in [3] and next have been extended to robust stability of fractional discrete-time linear systems in [4]. The stability of positive continuous-time linear systems with delays has been addressed in [5]. The independence of the asymptotic stability of positive 2D linear systems with delays of the number and values of the delays has been shown in [6]. The asymptotic stability of positive 2D linear systems without and with delays has been considered in $[7,8]$. The stability and stabilization of positive fractional linear systems by state-feedbacks have been analyzed in $[9,10]$. The Hurwitz stability of Metzler matrices has been investigated in [11] and some new tests for checking the asymptotic stability of positive $1 \mathrm{D}$ and $2 \mathrm{D}$ linear systems have been proposed in [12].

In this paper new tests for checking asymptotic stability of positive 1D continuous-time and discrete-time linear

\footnotetext{
*This work was supported by Ministry of Science and Higher Education in Poland under work No. N N514 638940.
}

systems without and with delays and of positive 2D linear systems described by the general and the Roesser models will be proposed. It will be shown that the checking of the asymptotic stability of positive 2D linear systems can be reduced to checking of suitable corresponding $1 \mathrm{D}$ positive linear systems.

The paper is organized as follows. In Section 2 new stability tests for positive continuous-time linear systems are proposed. An extension of these tests for positive discrete-time linear systems is given in Section 3. Application of these tests to checking the asymptotic stability of positive 1D linear systems with delays is given in Section 4. In Section 5 the tests are applied to positive 2D linear systems described by the general and Roesser models and in Section 6 the fractional discrete-time linear systems with delays. Concluding remarks are given in Section 7.

The following notation will be used: $\mathfrak{R}$-the set of real numbers, $\mathfrak{R}^{n \times m}$ - the set of $n \times m$ real matrices, $\mathfrak{R}_{+}^{n \times m}$ - the set of $n \times m$ matrices with nonnegative entries and $\Re_{+}^{n}=\Re_{+}^{n \times 1}, M_{n}$-the set of $n \times n$ Metzler matrices (real matrices with nonnegative off-diagonal entries), $I_{n}$ - the $n \times n$ identity matrix.

\section{Continuous-Time Linear Systems}

Consider the continuous-time linear system

$$
\dot{x}(t)=A x(t)
$$

where $x(t) \in \mathfrak{R}^{n}$ is the state vector and $A \in \mathfrak{R}^{n \times n}$.

The system (2.1) is called (internally) positive if 
$x(t) \in \mathfrak{R}_{+}^{n}, t \geq 0$ for any initial conditions

$x(0)=x_{0} \in \mathfrak{R}_{+}^{n}[1,2]$.

Theorem 2.1. [1,2] The system (2.1) is positive if and only if $A$ is a Metzler matrix.

The positive system is called asymptotically stable if

$$
\lim _{t \rightarrow \infty} x(t)=\lim _{t \rightarrow \infty} e^{A t} x_{0}=0 \text { for all } x_{0} \in \mathfrak{R}_{+}^{n}
$$

Theorem 2.2. [1,2] The positive system (2.1) is asymptotically stable if and only if all principal minors $\Delta_{i}, \quad i=1, \cdots, n$ of the matrix $-A$ are positive, i.e.

$$
\begin{aligned}
& \Delta_{1}=-a_{11}>0, \\
& \Delta_{2}=\left|\begin{array}{ll}
-a_{11} & -a_{12} \\
-a_{21} & -a_{22}
\end{array}\right|>0, \\
& \vdots \\
& \Delta_{n}=\operatorname{det}[-A]>0
\end{aligned}
$$

Theorem 2.3. [1,2] The positive system (2.1) is asymptotically stable only if all diagonal entries of the matrix $A$ are negative.

Let $A=\left[a_{i j}\right] \in \mathfrak{R}^{n \times n}$ be a Metzler matrix with negative diagonal entries $\left(a_{i i}<0, \quad i=1, \cdots, n\right)$. Let define

$$
\begin{aligned}
& A_{n}^{(0)}=A=\left[\begin{array}{ccc}
a_{11}^{(0)} & \ldots & a_{1, n}^{(0)} \\
\vdots & \ldots & \vdots \\
a_{n, 1}^{(0)} & \ldots & a_{n, n}^{(0)}
\end{array}\right]=\left[\begin{array}{cc}
a_{11}^{(0)} & b_{n-1}^{(0)} \\
c_{n-1}^{(0)} & A_{n-1}^{(0)}
\end{array}\right], \\
& A_{n-1}^{(0)}=\left[\begin{array}{ccc}
a_{22}^{(0)} & \ldots & a_{2, n}^{(0)} \\
\vdots & \ldots & \vdots \\
a_{n, 2}^{(0)} & \ldots & a_{n, n}^{(0)}
\end{array}\right], \\
& b_{n-1}^{(0)}=\left[\begin{array}{lll}
a_{12}^{(0)} & \ldots & a_{1, n}^{(0)}
\end{array}\right], \quad c_{n-1}^{(0)}=\left[\begin{array}{c}
a_{21}^{(0)} \\
\vdots \\
a_{n, 1}^{(0)}
\end{array}\right]
\end{aligned}
$$

and

$$
\begin{aligned}
A_{n-k}^{(k)} & =A_{n-k}^{(k-1)}-\frac{c_{n-k}^{(k-1)} b_{n-k}^{(k-1)}}{a_{k+1, k+1}^{(k-1)}}=\left[\begin{array}{ccc}
a_{k+1, k+1}^{(k)} & \ldots & a_{k+1, n}^{(k)} \\
\vdots & \ldots & \vdots \\
a_{n, k+1}^{(k)} & \ldots & a_{n, n}^{(k)}
\end{array}\right] \\
& =\left[\begin{array}{cc}
a_{k+1, k+1}^{(k)} & b_{n-k-1}^{(k)} \\
c_{n-k-1}^{(k)} & A_{n-k-1}^{(k)}
\end{array}\right], \\
A_{n-k-1}^{(k)} & =\left[\begin{array}{ccc}
a_{k+2, k+2}^{(k)} & \ldots & a_{k+2, n}^{(k)} \\
\vdots & \ldots & \vdots \\
a_{n, k+2}^{(k)} & \ldots & a_{n, n}^{(k)}
\end{array}\right], \\
b_{n-k-1}^{(k)} & =\left[\begin{array}{lll}
a_{k+1, k+2}^{(k)} & \ldots & a_{k+1, n}^{(k)}
\end{array}\right], \quad c_{n-k-1}^{(k)}=\left[\begin{array}{c}
a_{k+2, k+1}^{(k)} \\
\vdots \\
a_{n, k+1}^{(k)}
\end{array}\right]
\end{aligned}
$$

for $k=1, \cdots, n-1$.

Let us denote by $R[i+j \times c]$ the following elementary column operation on the matrix $A$ : addition to the $i$-th column the $j$-th column multiplied by a scalar $c$. It is well-known that using these elementary operations we may reduce the matrix

$$
A=\left[\begin{array}{cccc}
a_{11} & a_{12} & \ldots & a_{1, n} \\
a_{21} & a_{22} & \ldots & a_{2, n} \\
\vdots & \vdots & \ldots & \vdots \\
a_{n, 1} & a_{n, 2} & \ldots & a_{n, n}
\end{array}\right]
$$

to the lower triangular form

$$
\tilde{A}=\left[\begin{array}{cccc}
\tilde{a}_{11} & 0 & \ldots & 0 \\
\tilde{a}_{21} & \tilde{a}_{22} & \ldots & 0 \\
\vdots & \vdots & \ddots & \vdots \\
\tilde{a}_{n, 1} & \tilde{a}_{n, 2} & \ldots & \tilde{a}_{n, n}
\end{array}\right] .
$$

The reduction of the matrix (2.4) to the form (2.5) is equivalent to postmultiplication of the matrix (2.4) by the upper triangular matrix of the elementary column operations of the form

$$
\begin{aligned}
R & =\left[\begin{array}{cccc}
1 & r_{12} & \ldots & r_{1, n} \\
0 & 1 & \ldots & r_{2, n} \\
\vdots & \vdots & \ddots & \vdots \\
0 & 0 & \ldots & 1
\end{array}\right] \\
\tilde{A} & =A R
\end{aligned}
$$

Note that to reduce to zero the entries $a_{12}, \cdots, a_{1 n}$ of the matrix (2.4) we postmultiply it by the matrix

$$
R_{1}=\left[\begin{array}{cccc}
1 & -\frac{a_{12}}{a_{11}} & \ldots & -\frac{a_{1, n}}{a_{11}} \\
0 & 1 & \ldots & 0 \\
\vdots & \vdots & \ddots & \vdots \\
0 & 0 & \ldots & 1
\end{array}\right]
$$

and we obtain

$$
\bar{A}=A R_{1}=\left[\begin{array}{cccc}
a_{11} & 0 & \ldots & 0 \\
a_{21} & \bar{a}_{22} & \ldots & \bar{a}_{2, n} \\
\vdots & \vdots & \ldots & \vdots \\
a_{n, 1} & \bar{a}_{n, 2} & \ldots & \bar{a}_{n, n}
\end{array}\right]
$$

where

$$
\begin{aligned}
& \bar{a}_{22}=a_{22}-\frac{a_{12} \cdot a_{21}}{a_{11}}, \cdots, \bar{a}_{2, n}=a_{2, n}-\frac{a_{21} \cdot a_{1, n}}{a_{11}}, \\
& \bar{a}_{n, 2}=a_{n, 2}-\frac{a_{n, 1} \cdot a_{12}}{a_{11}}, \cdots, \bar{a}_{n, n}=a_{n, n}-\frac{a_{n, 1} \cdot a_{1, n}}{a_{11}} .
\end{aligned}
$$

Next we postmultiply the matrix (2.9) by the matrix

$$
R_{2}=\left[\begin{array}{ccccc}
1 & 0 & 0 & \ldots & 0 \\
0 & 1 & -\frac{\bar{a}_{23}}{\bar{a}_{22}} & \ldots & -\frac{\bar{a}_{2, n}}{\bar{a}_{22}} \\
0 & 0 & 1 & \ldots & 0 \\
\vdots & \vdots & \vdots & \ddots & \vdots \\
0 & 0 & 0 & \ldots & 1
\end{array}\right] .
$$


In a similar way we define matrices $R_{3}, \cdots, R_{n}$. The matrix (2.6) is the product of the elementary column operations matrices $R_{1}, R_{2}, \cdots, R_{n}$, i.e. $R=R_{1}, R_{2}, \cdots, R_{n}$.

It is easy to show that if the matrix (2.4) is Metzler matrix with negative diagonal entries then the matrix (2.5) is also a Metzler matrix.

Theorem 2.4. The positive systems with the matrix (2.5) is asymptotically stable if and only if all diagonal entries of the matrix are negative.

Proof. The eigenvalues of the matrix (2.5) are equal to its diagonal entries $\tilde{a}_{11}, \cdots, \tilde{a}_{n n}$ and the positive system is asymptotically stable if and only if all the diagonal entries are negative.

Theorem 2.5. The positive continuous-time linear system (2.1) is asymptotically stable if and only if one of the equivalent conditions is satisfied:

1) the diagonal entries of the matrices defined by (2.3)

$$
A_{n-k}^{(k)} \text { for } k=1, \cdots, n-1
$$

are negative,

2) the diagonal entries of the lower triangular matrix (2.5) are negative, i.e.

$$
\tilde{a}_{k k}<0 \text { for } k=1, \cdots, n
$$

Proof. Let $A_{j_{1}, \cdots, j_{q}}^{i_{1}, \cdots, i_{q}}$ be the $q \times q(q \leq n)$ minor of the matrix $A$ obtained by the deleting all rows except the rows $i_{1}, \cdots, i_{q}$ and all columns except the columns $j_{1}, \cdots, j_{q}$. In a similar way we define the minors of the matrices $\tilde{A}$ and $R$. Applying the Cauchy-Binet theorem to (2.7) we obtain

$$
\tilde{A}_{i_{1}, \cdots, i_{q}}^{i_{1}, \cdots, i_{q}}=\sum_{1 \leq k_{1}<\cdots<k_{q} \leq n} A_{k_{1}, \cdots, k_{q}}^{i_{1}, \cdots, i_{q}} R_{i_{1}, \cdots, i_{q}}^{k_{1}, \cdots, k_{q}}
$$

From the structure of the matrix (2.6) it follows that

$$
R_{i_{1}, \cdots, i_{q}}^{k_{1}, \cdots, k_{q}}=\left\{\begin{array}{l}
1 \text { for } k_{1}=i_{1}, \cdots, k_{q}=i_{q} \\
0 \text { for } k_{1} \neq i_{1}, \cdots, k_{q} \neq i_{q}
\end{array}\right.
$$

Taking into account (2.14) from (2.13) we obtain

$$
\tilde{A}_{i_{1}, \cdots, i_{q}}^{i_{1}, \cdots, i_{q}}=A_{i_{1}, \cdots, i_{q}}^{i_{1}, \cdots, i_{q}} \text { for } q=1, \cdots, n
$$

From (2.15) follows the equivalence of the conditions (2.2) and (2.11). To show the equivalence of the conditions (2.11) and (2.12) note that the computation of the matrix $A_{n-1}^{(1)}$ by the use of (2.3b) for $k=1$ is equivalent to the reduction to zero of the entries $a_{i, j}, j=2, \cdots, n$ of the matrix (2.4) by elementary column operations since

$$
A_{n-1}^{(1)}=\left[\begin{array}{ccc}
a_{22} & \ldots & a_{2, n} \\
\vdots & \ldots & \vdots \\
a_{n, 2} & \ldots & a_{n, n}
\end{array}\right]-\frac{1}{a_{11}}\left[\begin{array}{c}
a_{21} \\
\vdots \\
a_{n, 1}
\end{array}\right]\left[\begin{array}{lll}
a_{12} & \ldots & a_{1, n}
\end{array}\right] .
$$

Note that $-\frac{a_{1, i}}{a_{11}}>0$ for $i=2, \cdots, n$ and $-\frac{a_{1, i} a_{i, 1}}{a_{11}}>0$ for $i=2, \cdots, n$ since $a_{11}<0$ and $a_{i, j} \geq 0$ for $i \neq j$.
Thus, the matrix $A_{n-1}^{(1)}$ is a Metzler matrix. Continuing this procedure after $n$ steps we obtain the Metzler lower triangular matrix (2.5). Therefore, the conditions (2.11) and (2.12) are equivalent.

Example 2.1. Consider the positive system (2.1) with the matrix

$$
A=\left[\begin{array}{ccc}
-2 & 1 & 0 \\
0 & -1 & 1 \\
1 & 1 & -2
\end{array}\right]
$$

Check the asymptotic stability using the conditions (2.11) and (2.12).

Using (2.3) for (2.17) we obtain

$$
\begin{aligned}
A_{2}^{(1)} & =A_{2}^{(0)}-\frac{b_{2}^{(0)} c_{2}^{(0)}}{a_{33}^{(0)}} \\
= & {\left[\begin{array}{cc}
-1 & 1 \\
1 & -2
\end{array}\right]+\frac{1}{2}\left[\begin{array}{l}
0 \\
1
\end{array}\right]\left[\begin{array}{ll}
1 & 0
\end{array}\right]=\left[\begin{array}{cc}
-1 & 1 \\
1.5 & -2
\end{array}\right] } \\
A_{1}^{(2)} & =A_{1}^{(1)}-\frac{b_{1}^{(1)} c_{1}^{(1)}}{a_{22}^{(1)}}=-2+\frac{1.5}{1}=-0.5 .
\end{aligned}
$$

The conditions (2.11) of Theorem 2.5 are satisfied and the positive system is asymptotically stable.

Using the elementary column operations to the matrix (2.17) we obtain

$$
\begin{aligned}
& A=\left[\begin{array}{ccc}
-2 & 1 & 0 \\
0 & -1 & 1 \\
1 & 1 & -2
\end{array}\right] \stackrel{R[2+1 \times 0.5]}{\longrightarrow}\left[\begin{array}{ccc}
-2 & 0 & 0 \\
0 & -1 & 1 \\
1 & 1.5 & -2
\end{array}\right] \\
& \stackrel{R[3+2 \times 1]}{\longrightarrow}\left[\begin{array}{ccc}
-2 & 0 & 0 \\
0 & -1 & 0 \\
1 & 1.5 & -0.5
\end{array}\right]
\end{aligned}
$$

The conditions (2.12) of Theorem 2.5 are also satisfied and the positive system is asymptotically stable.

\section{Discrete-Time Linear Systems}

Consider the discrete-time linear system

$$
x_{i+1}=\bar{A} x_{i}, \quad i \in Z_{+}=\{0,1, \cdots\}
$$

where $x_{i} \in \mathfrak{R}^{n}$ is the state vector and $\bar{A} \in \mathfrak{R}^{n \times n}$.

The system (3.1) is called (internally) positive if $x_{i} \in \mathfrak{R}_{+}^{n}, \quad i \in Z_{+}$for any initial conditions $x_{0} \in \mathfrak{R}_{+}^{n}$.

Theorem 3.1. [1,2] The system (3.1) is positive if and only if $A \in \mathfrak{R}_{+}^{n \times n}$.

The positive system is called asymptotically stable if

$$
\lim _{i \rightarrow \infty} x_{i}=\lim _{i \rightarrow \infty} A^{i} x_{0}=0 \text { for all } x_{0} \in \mathfrak{R}_{+}^{n} .
$$

From Theorem 2.2 and 3.1 it follows that the nonnegative matrix $\bar{A}$ is asymptotically stable if and only if the Metzler matrix $\bar{A}-I_{n}$ is asymptotically stable.

Theorem 3.2. [1,2] The positive system (3.1) is as- 
ymptotically stable if and only if all principal minors $\hat{\Delta}_{i}, \quad i=1, \cdots, n$ of the matrix $\hat{A}=I_{n}-\bar{A}=\left[\hat{a}_{i j}\right] \in \mathfrak{R}^{n \times n}$ are positive, i.e.

$$
\hat{\Delta}_{1}=\hat{a}_{11}>0, \quad \hat{\Delta}_{2}=\left|\begin{array}{ll}
\hat{a}_{11} & \hat{a}_{12} \\
\hat{a}_{21} & \hat{a}_{22}
\end{array}\right|>0, \cdots, \quad \hat{\Delta}_{n}=\operatorname{det}[\hat{A}]>0 .
$$

Theorem 3.3. [1,2] The positive system (3.1) is asymptotically stable only if all diagonal entries of the matrix $\bar{A}$ are less than 1 .

It is assumed that $\bar{a}_{i i}<1, i=1, \cdots, n$ of the matrix $\bar{A}=\left[\bar{a}_{i j}\right] \in \mathfrak{R}_{+}^{n \times n}$ since otherwise by Theorem 3.3 the system is unstable. Using (2.3) in a similar way as for the matrix $A$ we define for the matrix $\hat{A}=\bar{A}-I_{n}=\left[\hat{a}_{i j}\right]$ the matrices $\hat{A}_{n-k}^{(k)}$ for $k=0,1, \cdots, n-1$. Using the elementary column operations we reduce the matrix $\hat{A}$ to the lower triangular form

$$
\tilde{A}^{\prime}=\left[\begin{array}{cccc}
\tilde{a}_{11}^{\prime} & 0 & \ldots & 0 \\
\tilde{a}_{21}^{\prime} & \tilde{a}_{22}^{\prime} & \ldots & 0 \\
\vdots & \vdots & \ddots & \vdots \\
\tilde{a}_{n, 1}^{\prime} & \tilde{a}_{n, 2}^{\prime} & \ldots & \tilde{a}_{n, n}^{\prime}
\end{array}\right] .
$$

Theorem 3.4. The positive discrete-time system with the matrix (3.4) is asymptotically stable if and only if all diagonal entries of the matrix $\hat{A}^{\prime}$ are less than 1 .

Proof is similar to the proof of Theorem 2.4.

Theorem 3.5. The positive discrete-time linear system (3.1) is asymptotically stable if and only if one of the equivalent conditions is satisfied:

1) the diagonal entries of the matrices

$$
\hat{A}_{n-k}^{(k)} \text { for } k=1, \cdots, n-1
$$

are negative,

2) the diagonal entries of the lower triangular matrix (3.4) are negative, i.e.

$$
\tilde{a}^{\prime}{ }_{k k}<0 \text { for } k=1, \cdots, n .
$$

Proof. The positive discrete-time system (3.1) is asymptotically stable if and only if the corresponding continuous-time system with the Metzler matrix $\hat{A}=\bar{A}-I_{n}$ is asymptotically stable. By Theorem 2.5 the positive discrete-time system (3.1) is asymptotically stable if one of its conditions is satisfied.

Example 3.1. Check the asymptotic stability of the positive system (3.1) with the matrix

$$
\bar{A}=\left[\begin{array}{ll}
0.5 & 0.1 \\
0.2 & 0.4
\end{array}\right] .
$$

In this case

$$
\hat{A}=\bar{A}-I_{n}=\left[\begin{array}{cc}
-0.5 & 0.1 \\
0.2 & -0.6
\end{array}\right] .
$$

Using (3.5) for $n=2$ we obtain

$$
\hat{A}_{1}^{(1)}=\hat{a}_{22}-\frac{\hat{a}_{12} \hat{a}_{21}}{\hat{a}_{11}}=-0.6+\frac{0.1 \cdot 0.2}{0.5}=-0.56<0 .
$$

Condition i) of Theorem 3.5 is satisfied and the positive system (3.1) with (3.7) is asymptotically stable.

Similarly, using the elementary column operations to the matrix (3.8) we obtain

$$
\hat{A}=\left[\begin{array}{cc}
-0.5 & 0.1 \\
0.2 & -0.6
\end{array}\right] \stackrel{R[2+1 \times 0.2]}{\longrightarrow}\left[\begin{array}{cc}
-0.5 & 0 \\
0.2 & -0.56
\end{array}\right] .
$$

The condition ii) of Theorem 3.5 is also satisfied and the positive system is asymptotically stable.

\section{Linear Systems with Delays}

Consider the continuous-time linear system with $q$ delays [5]

$$
\dot{x}(t)=A_{0} x(t)+\sum_{k=1}^{q} A_{k} x\left(t-d_{k}\right)
$$

where $x(t) \in \mathfrak{R}^{n}$ is the state vector, $A_{k} \in \mathfrak{R}^{n \times n}, \quad k=0,1, \cdots, q \quad$ and $\quad d_{k}>0, \quad k=1, \cdots, q \quad$ are delays.

The initial conditions for (4.1) have the form

$$
x(t)=x_{0}(t) \text { for } t \in[-d, 0], d=\max _{k} d_{k} .
$$

The system (4.1) is called (internally) positive if $x(t) \in \mathfrak{R}_{+}^{n}, t \geq 0$ for any initial conditions $x_{0}(t) \in \mathfrak{R}_{+}^{n}$.

Theorem 4.1. The system (4.1) is positive if and only if

$$
A_{0} \in M_{n} \text { and } A_{k} \in \Re_{+}^{n \times n}, k=1, \cdots, q
$$

where $M_{n}$ is the set of $n \times n$ Metzler matrices.

Proof is given in [5].

Theorem 4.2. The positive system with delays (4.1) is asymptotically stable if and only if the positive system without delays

$$
\dot{x}=A x, \quad A=\sum_{k=0}^{q} A_{k} \in M_{n}
$$

is asymptotically stable.

Proof is given in [5].

To check the asymptotic stability of the system (4.1) Theorem 2.5 is recommended. The application of Theorem 2.5 to checking the asymptotic stability of the system (4.1) will be demonstrated on the following example.

Example 4.1. Consider the system (4.1) with $q=1$ and the matrices

$$
A_{0}=\left[\begin{array}{cc}
-1 & 0.2 \\
0.2 & -1.4
\end{array}\right], \quad A_{1}=\left[\begin{array}{ll}
0.5 & 0.1 \\
0.2 & 0.8
\end{array}\right] .
$$

The matrix of the positive system (4.4) without delays has the form 


$$
A=A_{0}+A_{1}=\left[\begin{array}{cc}
-0.5 & 0.3 \\
0.4 & -0.6
\end{array}\right] \in M_{2} .
$$

Using (2.3) for the matrix (4.6) we obtain

$$
\hat{A}_{1}^{(1)}=-0.6+\frac{0.4 \cdot 0.3}{0.5}=-0.36<0 .
$$

Condition i) of Theorem 2.5 is satisfied and the positive system (4.1) with (4.5) is asymptotically stable.

Using the elementary column operations to the matrix (4.6) we obtain

$$
A=\left[\begin{array}{cc}
-0.5 & 0.3 \\
0.4 & -0.6
\end{array}\right] \stackrel{{ }^{[}\left[2+1 \times \frac{3}{5}\right]}{\longrightarrow}\left[\begin{array}{cc}
-0.5 & 0 \\
0.4 & -0.36
\end{array}\right]
$$

The condition ii) of Theorem 2.5 is also satisfied and the positive system is asymptotically stable.

Now let us consider the discrete-time linear system with $q$ delays [3]

$$
x_{i+1}=\sum_{k=0}^{q} A_{k} x_{i-k}, \quad i \in Z_{+}
$$

where $x_{i} \in \mathfrak{R}^{n}$ is the state vector and $A_{k} \in \mathfrak{R}^{n \times n}, k=$ $0,1, \cdots, q$.

The initial conditions for (4.8) have the form

$$
x_{-k} \in \mathfrak{R}^{n} \text { for } k=0,1, \cdots, q .
$$

The system (4.8) is called (internally) positive if $x_{i} \in \mathfrak{R}_{+}^{n}, \quad i \in Z_{+}$for any initial conditions $x_{-k} \in \mathfrak{R}_{+}^{n}$ for $k=0,1, \cdots, q$.

Theorem 4.3. [2] The system (4.8) is positive if and only if $A_{k} \in \mathfrak{R}_{+}^{n \times n}, k=0,1, \cdots, q$.

Theorem 4.4. The positive discrete-time system with delays (4.8) is asymptotically stable if and only if the positive system without delays

$$
x_{i+1}=\bar{A} x_{i}, \quad \bar{A}=\sum_{k=0}^{q} A_{k}, \quad i \in Z_{+}
$$

is asymptotically stable.

Proof is given in [3].

To check the asymptotic stability of the system (4.8) Theorem 3.5 is recommended. The application of Theorem 3.5 to checking the asymptotic stability of the system (4.8) will be demonstrated on the following example.

Example 4.2. Consider the positive system (4.8) with $q$ $=1$ and the matrices

$$
A_{0}=\left[\begin{array}{ll}
0.2 & 0.2 \\
0.1 & 0.2
\end{array}\right], \quad A_{1}=\left[\begin{array}{ll}
0.2 & 0.1 \\
0.1 & 0.3
\end{array}\right] .
$$

The matrix of the positive system (4.10) without delays has the form

$$
\bar{A}=A_{0}+A_{1}=\left[\begin{array}{ll}
0.4 & 0.3 \\
0.2 & 0.5
\end{array}\right] .
$$

In this case

$$
\hat{A}=\bar{A}-I_{n}=\left[\begin{array}{cc}
-0.6 & 0.3 \\
0.2 & -0.5
\end{array}\right]
$$

and using the elementary column operation to (4.13) we obtain

$$
\left[\begin{array}{cc}
-0.6 & 0.3 \\
0.2 & -0.5
\end{array}\right] \stackrel{R[2+1 \times 0.5]}{\longrightarrow}\left[\begin{array}{cc}
-0.6 & 0 \\
0.2 & -0.4
\end{array}\right] .
$$

The condition ii) of Theorem 3.5 is satisfied and the positive system is asymptotically stable.

\section{2D Linear Systems}

Consider the general autonomous model of 2D linear systems

$$
x_{i+1, j+1}=A_{0} x_{i, j}+A_{1} x_{i+1, j}+A_{2} x_{i, j+1}, \quad i, j \in Z_{+}
$$

where $x_{i, j} \in \mathfrak{R}^{n}$ is the state vector and $A_{k} \in \mathfrak{R}^{n \times n}, k=$ $0,1,2$.

Boundary conditions for (5.1) have the form

$$
x_{i, 0} \in \mathfrak{R}^{n}, i \in Z_{+} \text {and } x_{0, j} \in \mathfrak{R}^{n}, \quad j \in Z_{+} .
$$

The model (5.1) is called (internally) positive if $x_{i, j} \in \mathfrak{R}_{+}^{n}, i, j \in Z_{+}$for any initial conditions $x_{i, 0} \in \mathfrak{R}_{+}^{n}$, $i \in Z_{+}, \quad x_{0, j} \in \mathfrak{R}_{+}^{n}, \quad j \in Z_{+}$.

Theorem 5.1. [2] The system (5.1) is positive if and only if

$$
A_{k} \in \Re_{+}^{n \times n}, k=0,1,2 .
$$

The Roesser autonomous model of $2 \mathrm{D}$ linear systems has the form [2]

$$
\left[\begin{array}{c}
x_{i+1, j}^{h} \\
x_{i, j+1}^{v}
\end{array}\right]=\left[\begin{array}{ll}
A_{11} & A_{12} \\
A_{21} & A_{22}
\end{array}\right]\left[\begin{array}{c}
x_{i, j}^{h} \\
x_{i, j}^{v}
\end{array}\right], i, j \in Z_{+}
$$

where $x_{i, j}^{h} \in \mathfrak{R}^{n_{1}}$ and $x_{i, j}^{v} \in \mathfrak{R}^{n_{2}}$ are the horizontal and vertical state vectors at the point $(i, j)$ and $A_{k, l} \in \mathfrak{R}^{n_{k} \times n_{l}}, k$, $l=1,2$.

Boundary conditions for (5.4) have the form

$$
x_{0, j}^{h} \in \mathfrak{R}^{n_{1}}, \quad j \in Z_{+} \text {and } x_{i, 0}^{v} \in \mathfrak{R}^{n_{2}}, i \in Z_{+} .
$$

The model (5.4) is called (internally) positive if $x_{i, j}^{h} \in \mathfrak{R}_{+}^{n_{1}}$ and $x_{i, j}^{v} \in \mathfrak{R}_{+}^{n_{2}}$ for any initial conditions $x_{0, j}^{h} \in \mathfrak{R}_{+}^{n_{1}}, \quad j \in Z_{+}$and $x_{i, 0}^{v} \in \mathfrak{R}_{+}^{n_{2}}, i \in Z_{+}$.

Theorem 5.2. [2] The Roesser model (5.4) is positive if and only if

$$
\left[\begin{array}{ll}
A_{11} & A_{12} \\
A_{21} & A_{22}
\end{array}\right] \in \mathfrak{R}_{+}^{n \times n}, \quad n=n_{1}+n_{2} .
$$

The positive general model (5.1) is called asymptotically stable if

$$
\lim _{i, j \rightarrow \infty} x_{i, j}=0 \text { for all } x_{i, 0} \in \mathfrak{R}_{+}^{n}, \quad i \in Z_{+}
$$


and

$$
x_{0, j} \in \mathfrak{R}_{+}^{n}, \quad j \in Z_{+} .
$$

Similarly, the positive Roesser model (5.4) is called asymptotically stable if

$$
\begin{gathered}
\lim _{i, j \rightarrow \infty}\left[\begin{array}{c}
x_{i, j}^{h} \\
x_{i, j}^{v}
\end{array}\right]=0 \text { for all } x_{0, j}^{h} \in \mathfrak{R}_{+}^{n_{1}}, \quad j \in Z_{+} \\
\text {and } x_{i, 0}^{v} \in \mathfrak{R}_{+}^{n_{2}}, \quad i \in Z_{+} .
\end{gathered}
$$

Theorem 5.3. The positive general model (5.1) is asymptotically stable if and only if the positive 1D system

$$
x_{i+1}=A x_{i}, \quad A=A_{0}+A_{1}+A_{2}, \quad i \in Z_{+}
$$

is asymptotically stable.

Proof is given in $[8,6]$.

Theorem 5.4. The positive Roesser model (5.4) is asymptotically stable if and only if the positive 1D system

$$
x_{i+1}=\left[\begin{array}{ll}
A_{11} & A_{12} \\
A_{21} & A_{22}
\end{array}\right] x_{i}, \quad i \in Z_{+}
$$

is asymptotically stable.

Proof is given in $[8,6]$.

To check the asymptotic stability of the positive general model (5.1) and the positive Roesser model (5.4) the Theorem 3.5 is recommended. The application of Theorem 3.5 to checking the asymptotic stability of the models (5.1) and (5.4) will be demonstrated on the following examples.

Example 5.1. Consider the positive general model (5.1) with the matrix

$$
A_{0}=\left[\begin{array}{ll}
0.1 & 0.2 \\
0.1 & 0.1
\end{array}\right], \quad A_{1}=\left[\begin{array}{ll}
0 & 0.1 \\
0 & 0.1
\end{array}\right], \quad A_{1}=\left[\begin{array}{ll}
0.2 & 0.3 \\
0.1 & 0.2
\end{array}\right] .
$$

In this case

$$
\bar{A}=A_{0}+A_{1}+A_{2}=\left[\begin{array}{ll}
0.3 & 0.6 \\
0.2 & 0.4
\end{array}\right]
$$

and

$$
\hat{A}=\bar{A}-I_{n}=\left[\begin{array}{cc}
-0.7 & 0.6 \\
0.2 & -0.6
\end{array}\right] .
$$

Using the elementary column operation to (5.13) we obtain

$$
\hat{A}=\left[\begin{array}{cc}
-0.7 & 0.6 \\
0.2 & -0.6
\end{array}\right] \stackrel{{ }^{[}\left[2+1 \times \frac{6}{7}\right]}{\longrightarrow}\left[\begin{array}{cc}
-0.7 & 0 \\
0.2 & -\frac{3}{7}
\end{array}\right] .
$$

The condition ii) of Theorem 3.5 is satisfied and the positive general model with (5.11) is asymptotically stable.

Example 5.2. Consider the positive Roesser model (5.4) with the matrices

$$
A=\left[\begin{array}{ll}
A_{11} & A_{12} \\
A_{21} & A_{22}
\end{array}\right]
$$

and

$$
\begin{aligned}
& A_{11}=\left[\begin{array}{ll}
0.6 & 0.2 \\
0.1 & 0.4
\end{array}\right], \quad A_{12}=\left[\begin{array}{l}
0.1 \\
0.2
\end{array}\right], \\
& A_{21}=\left[\begin{array}{ll}
0.2 & 0.1
\end{array}\right], \quad A_{22}=[0.8] .
\end{aligned}
$$

In this case

$$
\hat{A}=A-I_{n}=\left[\begin{array}{ccc}
-0.4 & 0.2 & 0.1 \\
0.1 & -0.6 & 0.2 \\
0.2 & 0.1 & -0.2
\end{array}\right] .
$$

Using the elementary column operation to (5.15) we obtain

$$
\begin{gathered}
{\left[\begin{array}{ccc}
-0.4 & 0.2 & 0.1 \\
0.1 & -0.6 & 0.2 \\
0.2 & 0.1 & -0.2
\end{array}\right] \stackrel{\substack{R[2+1 \times 0.5] \\
R[3+1 \times 0.25]}}{\longrightarrow}\left[\begin{array}{ccc}
-0.4 & 0 & 0 \\
0.1 & -0.55 & 0.225 \\
0.2 & 0.2 & -0.15
\end{array}\right]} \\
\stackrel{R\left[3+2 \times \frac{22.5}{55}\right]}{\longrightarrow}\left[\begin{array}{ccc}
-0.4 & 0 & 0 \\
0.1 & -0.55 & 0 \\
0.2 & 0.2 & -0.0682
\end{array}\right]
\end{gathered}
$$

The condition ii) of Theorem 3.5 is satisfied and the positive Roesser model with (5.14) is asymptotically stable.

In a similar way as for 1D linear systems using the approach given in [7] the considerations can be easily extended to 2D linear systems with delays and to fractional 1D and 2D linear systems.

\section{Fractional Positive Discrete-Time Linear Systems}

Consider the autonomous fractional discrete-time linear systems with $q$ delays [10]

$$
X_{i+1}=\sum_{j=1}^{i+1}(-1)^{j+1}\left(\begin{array}{c}
\alpha \\
j
\end{array}\right) x_{i-j+1}+\sum_{k=0}^{q} A_{k} x_{i-k}, \quad 0<\alpha<1
$$

where $\alpha$ is the fractional order, $x_{i} \in \mathfrak{R}^{n}$ is the state vector, $A_{k} \in \mathfrak{R}^{n \times n}, k=0,1, \cdots, q$ and

$$
\left(\begin{array}{c}
\alpha \\
j
\end{array}\right)=\left\{\begin{array}{cc}
1 & \text { for } \quad j=0 \\
\frac{\alpha(\alpha-1) \ldots(\alpha-j+1)}{j !} & \text { for } j=1,2, \cdots
\end{array}\right.
$$

The fractional system (6.1) is called (internally) positive if $x_{i} \in \mathfrak{R}_{+}^{n}$, for any initial conditions $x_{-k} \in \mathfrak{R}_{+}^{n}$, $k=0,1, \cdots, q$.

Theorem 6.1. [10] The fractional system (6.1) is positive if and only if $A_{k}+c_{k+1} I_{n} \in \mathfrak{R}_{+}^{n \times n}$ for $k=0,1, \cdots, q$ 
where $c_{k}=(-1)^{k}\left(\begin{array}{l}\alpha \\ k\end{array}\right)$.

The fractional positive system (6.1) is called asymptotically stable if

$$
\lim _{i \rightarrow \infty} x_{i}=0 \text { for all } x_{-k} \in \mathfrak{R}_{+}^{n}, \quad k=0,1, \cdots, q .
$$

Theorem 6.2. [10] The fractional positive system (6.1) is asymptotically stable if and only if the positive discrete-time system without delays

$$
x_{i+1}=\bar{A} x_{i}, \quad \bar{A}=-I_{n}+\sum_{k=0}^{q} A_{k}
$$

is asymptotically stable.

Proof is given in [10].

To check the asymptotic stability of the fractional positive system (6.1) Theorem 3.5 is recommended. The application of Theorem 3.5 to checking the asymptotic stability of the system (6.1) will be demonstrated on the following example.

Example 6.1. Consider the fractional system (6.1) for $\alpha=0.5$ with $q=1$ and the matrices

$$
A_{0}=\left[\begin{array}{ll}
0.55 & 0.1 \\
0.05 & 0.5
\end{array}\right], \quad A_{1}=\left[\begin{array}{cc}
0.2 & 0.1 \\
0.05 & 0.2
\end{array}\right] .
$$

The fractional system is positive since

$$
A_{0}+c_{1} I_{2}=A_{0}-\alpha I_{2}=\left[\begin{array}{cc}
0.05 & 0.1 \\
0.05 & 0
\end{array}\right] \in \mathfrak{R}_{+}^{2 \times 2}
$$

and

$$
A_{1}+c_{2} I_{2}=A_{2}+\frac{\alpha(\alpha-1)}{2} I_{2}=\left[\begin{array}{cc}
0.075 & 0.1 \\
0.05 & 0.075
\end{array}\right] \in \mathfrak{R}_{+}^{2 \times 2}
$$

Therefore, to check the asymptotic stability of the positive system we may use Theorem 3.5.

Using (3.5) for $n=2$ and (6.4) we obtain

$$
\hat{A}=\bar{A}-I_{2}=A_{0}+A_{1}-2 I_{2}=\left[\begin{array}{cc}
-1.25 & 0.2 \\
0.1 & -1.3
\end{array}\right]
$$

and

$$
\hat{A}_{1}^{(1)}=\hat{a}_{22}+\frac{\hat{a}_{12} \hat{a}_{21}}{\hat{a}_{11}}=-1.3+\frac{0.2 \cdot 0.1}{1.25}=-1.284<0 .
$$

The condition i) of Theorem 3.5 is also satisfied and the positive system is asymptotically stable.

Using the elementary column operations to the matrix (6.7) we obtain

$$
\left[\begin{array}{cc}
-1.25 & 0.2 \\
0.1 & -1.3
\end{array}\right] \stackrel{R[2+1 \times 0.16]}{\longrightarrow}\left[\begin{array}{cc}
-1.25 & 0 \\
0.1 & -1.284
\end{array}\right] .
$$

The condition ii) of Theorem 3.5 is satisfied and the positive system is asymptotically stable.

This approach can be also applied to checking the asymptotic stability of the positive $2 \mathrm{D}$ linear systems with delays.

\section{Concluding Remarks}

New tests for checking asymptotic stability of positive 1D continuous-time and discrete-time linear systems without and with delays and of positive 2D linear systems described by the general and the Roesser models have been proposed. The tests are based on the Theorem 2.5 and Theorem 3.5. Checking of the asymptotic stability of positive 2D linear systems has been reduced to checking of suitable corresponding 1D positive linear systems. It has been shown that the stability tests can be also applied to checking the asymptotic stability of fractional discrete-time linear systems with delay. The tests can be also extended to 2D continuous-discrete linear systems and to $1 \mathrm{D}$ and 2D fractional linear systems. An open problem is extension of these considerations to 2D positive switched linear systems.

\section{References}

[1] L. Farina and S. Rinaldi, "Positive Linear Systems; Theory and Applications," John Wiley \& Sons, Hoboken, 2000. doi:10.1002/9781118033029

[2] T. Kaczorek, "Positive 1D and 2D Systems," Springer Verlag, London, 2002. doi:10.1007/978-1-4471-0221-2

[3] M. Busłowicz, "Simple Stability Conditions for Linear Systems with Delays," Bulletin of the Polish Academy of Sciences, Vol. 56, No. 4, 2008, 319-324.

[4] M. Busłowicz, "Robust Stability of Positive DiscreteTime Linear Systems of Fractional Order," Bulletin of the Polish Academy of Sciences, Vol. 58, No. 4, 2010, 567-572. doi:10.2478/v10175-010-0057-8

[5] T. Kaczorek, "Stability of Positive Continuous-Time Linear Systems with Delays," Bulletin of the Polish Academy of Sciences, Vol. 57, No. 4, 2009, 395-398. doi:10.2478/v10175-010-0143-y

[6] T. Kaczorek, "Independence of Asymptotic Stability of Positive 2D Linear Systems with Delays of Their Delays," International Journal of Applied Mathematics and Computer Science, Vol. 19, No. 2, 2009, 255-261. doi:10.2478/v10006-009-0021-7

[7] T. Kaczorek, "Asymptotic Stability of Positive 2D Linear Systems with Delays," Bulletin of the Polish Academy of Sciences, Vol. 57, No. 2, 2009, 133-138. doi:10.2478/v10175-010-0113-4

[8] T. Kaczorek, "Asymptotic Stability of Positive 2D Linear Systems," Computer Applications in Electrical Engineering, Poznan University of Technology, Electrical Engineering Committee of Polish Academy of Sciences, IEEE Poland Section, Poznan. 
[9] T. Kaczorek, "Stability and Stabilization of Positive Fractional Linear Systems by State-Feedbacks," Bulletin of the Polish Academy of Sciences, Vol. 58, No. 4, 2010, 517-554.

[10] T. Kaczorek, "Selected Problems of Fractional System Theory," Springer Verlag, London, 2011. doi:10.1007/978-3-642-20502-6
[11] K. S. Narendra and R. Shorten, "Hurwitz Stability of Metzler Matrices," IEEE Transactions on Automatic Control, Vol. 55, no. 6 June 2010, pp. 1484-1487. doi:10.1109/TAC.2010.2045694

[12] T. Kaczorek, "New Stability Tests of Positive 1D and 2D Linear Systems," Proceeding of 25th European Conference Modelling and Simulation, Krakow, 7-10 June 2011. 